\title{
Superconducting Proximity Effect through a Magnetic Domain Wall
}

\author{
Alexander Konstandin ${ }^{1}$, Juha Kopu ${ }^{1,2}$, and Matthias Eschrig ${ }^{1}$, \\ ${ }^{1}$ Institut für Theoretische Festkörperphysik, Universität Karlsruhe, 76128 Karlsruhe, Germany \\ ${ }^{2}$ Low Temperature Laboratory, Helsinki University of Technology, FIN-02015 HUT, Finland
}

(Dated: July 20, 2005)

\begin{abstract}
We study the superconducting proximity effect in a superconductor-ferromagnet-superconductor (SFS) heterostructure, containing a domain wall in the ferromagnetic region. For the ferromagnet we assume an alloy with an exchange splitting of the conduction bands comparable to the superconducting gaps. We calculate the modification of the density of states in the center of the domain wall as a result of the proximity effect. We show that the density of states is sensitive to domain wall parameters due to triplet-pairing correlations created in vicinity of the domain wall. We present a theoretical tool which in a very effective way enables retaining the full spatially dependent spin-space structure of the problem.
\end{abstract}

PACS numbers: $74.45 .+\mathrm{c}, 74.50 .+\mathrm{r}$

Introduction: Most promising candidates for mesoscopic devices with novel functionality are hybrid structures containing superconducting elements. The key phenomenon that controls the behavior of such systems is the proximity effect. When a superconducting material is placed in contact with a normal metal $(\mathrm{N})$, the superconducting pair correlations leak over to the normal-metal side, changing its conduction properties in the vicinity of the separating interface. Quite similarly, the properties on the superconducting side are also changed (the energy gap $\Delta_{0}$ is suppressed) due to the contact to a normal metal. An alternative but equivalent way of thinking about the proximity effect is through Andreev-reflection 1 processes: an incoming electron from the normal side is transmitted together with another one as a Cooper pair into the superconducting side. This phase-coherent electron-hole conversion results in a nonzero pair amplitude in the normal metal.

In the diffusive limit, the correlations relating to an incident electron with an energy $E$ (the range of energies being set by the temperature $T$ ) above the chemical potential extend a characteristic distance of $\xi_{N}=\sqrt{D / E}$ into the normal metal, ${ }^{2}$ here $D$ is the diffusion constant in $\mathrm{N}$. If the extent of the $\mathrm{N}$ region is finite, another energy scale, $E_{T} \sim D / L^{2}$, enters the problem; $L$ denotes the width of N. This so-called Thouless energy has associated with it one of the generic features of diffusive superconductor-normal metal heterostructures, the minigap: the density of states in the normal metal develops a gap around the chemical potential in a manner similar to a superconductor ( $\mathrm{S}$ ) but with a smaller magnitude.

If the normal conductor is replaced by a ferromagnet (F), a multitude of new effects arise due to the emergence of yet another energy scale, that of the exchange splitting $J$ of the two spin bands. Both on the theoretical 3.4.5.6.7.8.9.10.11 and on

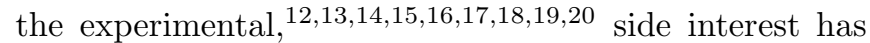
grown recently in the rich physics of such systems. One source for new behavior is that, in the case with a singlet superconductor, the induced pair amplitude in the ferromagnet is oscillatory ${ }^{21}$ However, the exchange splitting also gives rise to dephasing which, in turn, results in the decay of induced correlations over a characteristic distance $\xi_{J}=\sqrt{D /(E+J)}$.22 Unfortunately, since $J$ is of the order of the Fermi energy $E_{F}$ in typical ferromagnetic metals, this distance is very short. Still, experimental indication of the oscillatory behavior has been obtained in thin ferromagnetic layers and, relevant to the present paper, in weakly ferromagnetic alloys with $J \ll E_{F}, \underline{12,13}$

Another question of current interest in SF proximity systems is the role of equal-spin triplet correlations ${ }^{23.24}$ If created, e.g. near magnetic inhomogeneities, such correlations would not be affected by the exchange splitting but could penetrate considerably longer distances into $\mathrm{F}, 23$ Finally, the importance of domain walls has also been stressed for the Andreev conductance ${ }^{25.26}$.

In this paper we study an SFS structure, shown schematically in Fig. 1 in equilibrium. The ferromagnetic region consists of two domains with magnetizations oriented in opposite directions. The domains are separated by a domain wall, where the magnetization rotates continuously between the asymptotic values. While varying in direction, the magnitude $J$ is assumed constant throughout the F region. We show, that the local density of states (LDOS) in the F region is strongly modified by the presence of the domain wall. In particular, we show that it can be very sensitive to the thickness of the

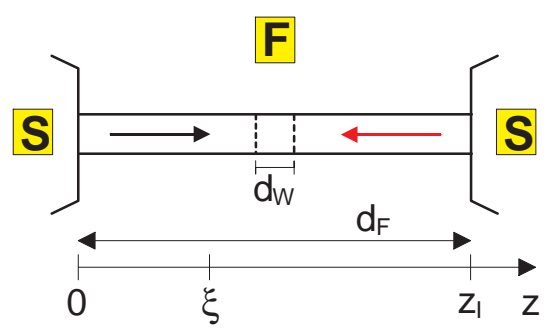

FIG. 1: SFS structure with two magnetic domains oriented along the $z$ axis and separated by a domain wall of width $d_{W}$; $d_{F}$ denotes the length of the $\mathrm{F}$ region and $\xi=\sqrt{D / \Delta_{0}}$ is the superconducting coherence length. 
domain wall in a certain parameter region.

Basic equations: Proximity effect is a spatially inhomogeneous phenomenon. An appropriate theoretical tool to treat such a problem is the quasiclassical theory of superconductivity ${ }_{27.28}^{27}$ which in its diffusive version has been formulated by Usadel ${ }^{29}$ In equilibrium, the physical information is contained in the retarded Green functions $\hat{G}(z, E)$. Here, we assume spatial dependence in the coordinate $z$ only, and $E$ denotes the energy as measured from the chemical potential. The $4 \times 4$ matrix structure, arising from particle-hole and spin degrees of freedom, is denoted by the hat $\left({ }^{\wedge}\right)$ accent,

$$
\hat{G}=\left(\begin{array}{cc}
\mathcal{G} & \mathcal{F} \\
\tilde{\mathcal{F}} & \tilde{\mathcal{G}}
\end{array}\right) .
$$

The off-diagonal elements determine the superconducting pair amplitude. Quantities denoted with the "tilde" are related to those without one through $\tilde{\mathcal{A}}(z, E)=$ $\mathcal{A}\left(z,-E^{*}\right)^{*}$. All the elements in Eq. (11) are $2 \times 2$ spin matrices: e.g. $\mathcal{G}=\mathcal{G}_{\alpha \beta}$ with $\alpha, \beta=\{\uparrow, \downarrow\}$. The Green functions satisfy the Usadel equation, ${ }^{29}$

$$
\left[E \hat{\tau}_{3}-\hat{\Delta}-\boldsymbol{J} \cdot \hat{\boldsymbol{\sigma}}, \hat{G}\right]_{\otimes}+\frac{D}{\pi} \frac{\mathrm{d}}{\mathrm{d} z}\left(\hat{G} \otimes \frac{\mathrm{d}}{\mathrm{d} z} \hat{G}\right)=\hat{0}
$$

where the symbol $\otimes$ denotes matrix multiplication, and $[\hat{A}, \hat{B}]_{\otimes}=\hat{A} \otimes \hat{B}-\hat{B} \otimes \hat{A}$. In writing Eq. (2) we have followed the standard way to describe ferromagnetic materials through a spin-dependent energy shift, ${ }^{22}$ which has the form $E \hat{\tau}_{3} \rightarrow E \hat{\tau}_{3}-\boldsymbol{J} \cdot \hat{\boldsymbol{\sigma}}$. Here, $\tau_{3}$ denotes the third Pauli-matrix in Nambu space, the vector $\boldsymbol{J}$ denotes the effective exchange field of the ferromagnet, and $\hat{\Delta}$ is the superconducting order parameter (appropriate for weakcoupling spin-singlet pairing). The components of the vector $\hat{\boldsymbol{\sigma}}$ and the order parameter are given by

$$
\hat{\sigma}_{i}=\left(\begin{array}{cc}
\sigma_{i} & 0 \\
0 & \sigma_{i}^{*}
\end{array}\right), \quad \hat{\Delta}=\left(\begin{array}{cc}
0 & \Delta \\
\Delta^{*} & 0
\end{array}\right)
$$

where $\sigma_{i}$ are Pauli spin matrices, $i=x, y, z$, and $\Delta=$ $\Delta_{0} i \sigma_{y}$. The above procedure is appropriate for describing situations for which $J \ll E_{F}$, which holds e.g. for the ferromagnetic alloys used in Refs. 12, 13. In writing Eq. (2), we have chosen the normalization according to

$$
\hat{G} \otimes \hat{G}=-\pi^{2} \hat{1} .
$$

Riccati parameterization: The spin-dependent nature of SF proximity systems calls for a formulation of the quasiclassical theory that retains the full spinspace structure, especially in studying situations where the exchange-field-orientation varies in space (such as in a domain wall). Within the Eilenberger theory a very convenient formulation already exists, 30 employing the so-called Riccati parameterization ${ }^{31.32}$ The extension of this method to the Usadel theory was achieved only recently, 33 and has been applied to non-equilibrium situations $\stackrel{34}{=}$ and to FSF-systems with homogeneous magnetizations $\stackrel{35}{5}$ Here we demonstrate its usefulness by applying it to a SFS system with a spatially changing magnetization in a domain wall, a case where the conventional so-called $\theta$-parameterization ${ }^{36}$ is not applicable. The spin-dependent Riccati parameterization,

$$
\hat{G}=-i \pi \hat{N} \otimes\left(\begin{array}{cc}
(1+\gamma \otimes \tilde{\gamma}) & 2 \gamma \\
-2 \tilde{\gamma} & -(1+\tilde{\gamma} \otimes \gamma)
\end{array}\right),
$$

with

$$
\hat{N}=\left(\begin{array}{cc}
(1-\gamma \otimes \tilde{\gamma})^{-1} & 0 \\
0 & (1-\tilde{\gamma} \otimes \gamma)^{-1}
\end{array}\right)
$$

automatically accounts for the normalization (4), which is essential for practical numerical calculations. It is enough to determine one $2 \times 2$ matrix in spin space, $\gamma$. The other, $\tilde{\gamma}$, follows from the above-mentioned (fundamental) symmetry. The transport equation for $\gamma$ follows from Eq. (2), and reads 33

$$
\begin{aligned}
\frac{\mathrm{d}^{2} \gamma}{\mathrm{d} z^{2}}+ & \left(\frac{\mathrm{d} \gamma}{\mathrm{d} z}\right) \otimes \frac{\tilde{\mathcal{F}}}{i \pi} \otimes\left(\frac{\mathrm{d} \gamma}{\mathrm{d} z}\right)=\frac{i}{D}\left[\gamma \otimes \Delta^{*} \otimes \gamma\right. \\
& \left.-(E-\boldsymbol{J} \cdot \boldsymbol{\sigma}) \otimes \gamma-\gamma \otimes\left(E+\boldsymbol{J} \cdot \boldsymbol{\sigma}^{*}\right)-\Delta\right],
\end{aligned}
$$

Here, the expression for $\tilde{\mathcal{F}}$ is obtained by comparing Eq. (11) with Eqs. (5)-(6).

Boundary conditions: Additionally, boundary conditions are required for the different interfaces of the system. Such conditions have been formulated by Nazarov ${ }^{37}$ The outer surfaces $\left(z=z_{o}\right)$ of the superconductors are assumed to border to an insulating region, and the appropriate condition is $\partial_{z} \hat{G}\left(z_{o}, E\right)=0$, i.e.

$$
\frac{d \gamma}{d z}\left(z_{o}, E\right)=0
$$

On the other hand, the two inner SF interfaces $\left(z=z_{i}^{S}\right.$ for the $\mathrm{S}$ side, $z=z_{i}^{F}$ for the $\mathrm{F}$ side) are assumed in the following transparent. The corresponding boundary conditions are in this case $\hat{G}\left(z_{i}^{S}, E\right)=\hat{G}\left(z_{i}^{F}, E\right)$, $\sigma_{S} \partial_{z} \hat{G}\left(z_{i}^{S}, E\right)=\sigma_{F} \partial_{z} \hat{G}\left(z_{i}^{F}, E\right)$, leading to

$$
\begin{aligned}
\gamma\left(z_{i}^{S}, E\right) & =\gamma\left(z_{i}^{F}, E\right), \\
\sigma_{S} \frac{d \gamma}{d z}\left(z_{i}^{S}, E\right) & =\sigma_{F} \frac{d \gamma}{d z}\left(z_{i}^{F}, E\right),
\end{aligned}
$$

where $\sigma_{S}$ and $\sigma_{F}$ refer to the conductivities of $\mathrm{S}$ and $\mathrm{F}$, respectively. For simplicity, we have assumed $\sigma_{S}=\sigma_{F}$, implying the continuity of the derivative at the interface. With the boundary conditions (8) and (9), we have solved Eq. (7) numerically by an iterative procedure (relaxation method) in the entire SFS system.

SFS system with domain wall: We apply the outlined theory to study the SFS structure of Fig. 1 in equilibrium. Lengths are given in units of the superconducting coherence length, $\xi=\sqrt{D / \Delta_{0}}$. The spin-singlet superconductors are chosen to have the same gap magnitude. The contact areas at the SF interfaces are assumed to 


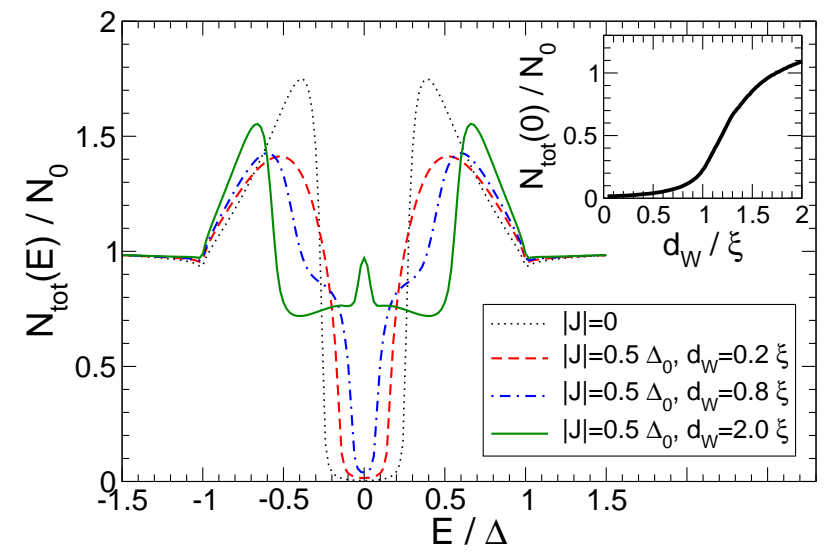

FIG. 2: LDOS as a function of energy for the system of Fig. 1] calculated in the middle of the $\mathrm{F}$ region for different widths $d_{W}$ of the domain wall. Here, $J=0.5 \Delta_{0}, d_{F}=2.0 \xi$. For comparison, the dotted line shows the corresponding dependence in the normal-metal case $(J=0)$. The inset shows for $J=0.5 \Delta_{0}$, the value of the LDOS at the chemical potential $(E=0)$ as a function of the domain-wall width $d_{W}$.

be small enough, so that any gap suppression can be neglected. The two superconducting regions and the intermediate ferromagnet are taken to have fixed lengths of $d_{S}=5 \xi$ and $d_{F}=2 \xi$. We model the domain wall by a varying direction of $\mathbf{J}=\left(J_{x}, J_{y}, J_{z}\right)$ (keeping the magnitude $J=|\mathbf{J}|$ constant), with $J_{y}=0$ and

$$
\left(\begin{array}{l}
J_{x} \\
J_{z}
\end{array}\right)=J\left(\begin{array}{c}
\cos \theta(z) \\
\sin \theta(z)
\end{array}\right), \theta(z)=-\arctan \frac{z-z_{0}}{d_{W}}
$$

Here, $d_{W}$ is an effective domain wall width parameter. In the following we study the influence of the width $d_{W}$ of a domain wall centered in $\mathrm{F}\left(z_{0}=d_{F} / 2\right)$ on the density of states in the center of the domain wall $\left(z=z_{0}\right)$.

Knowing $\gamma(z, E)$ from the solution of Eq. (7) with boundary conditions (8) and (9), the quasiclassical Green function and the (total) LDOS

$$
N_{t o t}(z, E)=-\frac{N_{0}}{2 \pi} \operatorname{Im} \operatorname{Tr} \mathcal{G}(z, E),
$$

is determined via Eqs. (5)-(6); $N_{0}$ is the normal-state density of states, and Tr denotes the spin trace.

An important characteristic of the value of the LDOS in superconductor-normal metal proximity systems is the minigap: the density of states in the normal-metal region shows a gap of width $E_{g}<\Delta_{0}$ induced by proximity to a superconductor. The energy $E_{g}$ can be thought of as that of the lowest-energy Andreev bound state in a finite normal-metal layer. This convenient physical picture can easily be extended to single-domain ferromagnets. The corresponding spin-dependent energy shift of the quasiparticle and the Andreev-reflected quasihole by $\pm J$ leads to a reduction of the energy of the lowest-lying bound state, and correspondingly of the minigap, from the expression for a normal metal by $J$, vanishing altogether

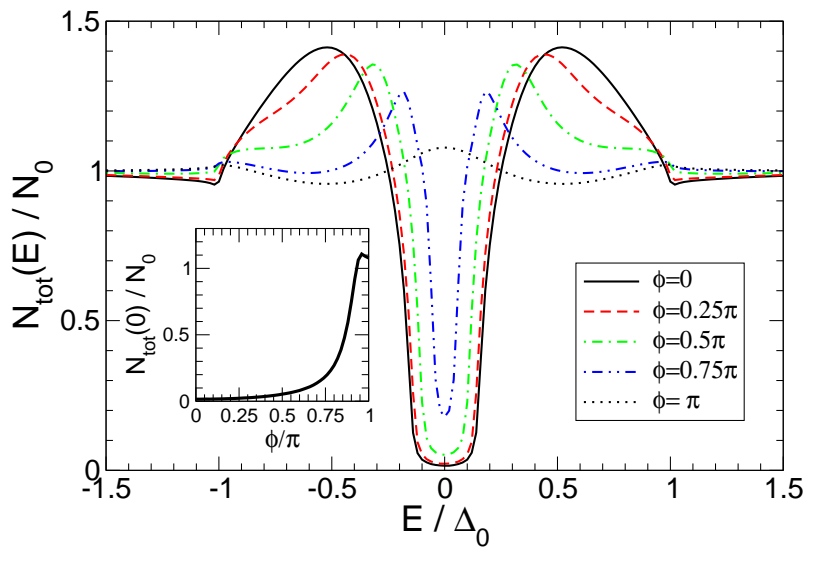

FIG. 3: LDOS as a function of energy for several phase differences $\phi$ between the superconductors, calculated in the middle of F. Here, $J=0.5 \Delta_{0}, d_{F}=2.0 \xi$. The width of the domain wall is $d_{W}=0.2 \xi$. The inset shows the corresponding LDOS at the chemical potential $(E=0)$ as a function of $\phi$.

when $J \geq E_{g, J=0}$. This picture is confirmed by our numerical calculations.

In the inhomogeneously magnetized case of Fig. 1] the above picture is modified. The effect of the domain wall on the LDOS is summarized in Fig. 2 which shows $N_{t o t}$ as a function of energy for different domain-wall widths $d_{W}$. Although the value of $J=0.5 \Delta_{0}$ is here larger than the value of the normal state minigap $E_{g} \approx 0.25 \Delta_{0}$ (as seen from the dotted curve in Fig. 2 for $J=0$ ), the minigap is reduced to zero only for larger domain wall widths $d_{W} \approx 2 \xi$. For the smallest width $d_{W}=$ $0.2 \xi$ the minigap is only reduced by about $40 \%$. The additional states which fill the minigap with increasing domain wall width are due to spin triplet correlations, which are sensitive to the direction of $\mathbf{J}$. Our calculations show that the influence of equal-spin pairing components created by the domain wall increases. This is reflected by the appearance of additional Andreev bound states inside the gap, modifying the LDOS. The relative importance of the triplet correlations depends on $J$ and $d_{W}$ : as clearly manifested by Fig. 2 the efficiency of the triplet-inducing mechanism grows with increasing $d_{W}$. The inset of Fig. 2 shows the value of the LDOS at $E=0$ as a function of $d_{W}$. The interesting observation here is that the LDOS at the chemical potential is very sensitive to the domain wall width when the latter one is comparable to $\xi$.

Finally, with a view towards studying the possible effects of the domain walls on the supercurrent flowing in an SFS structure, we have studied the LDOS in the case where there is a phase difference $\phi$ between the two superconductors. This phase difference adds to the one accumulated by the quasiparticles and quasiholes in the ferromagnetic region and, thus, modifies the spectrum of Andreev bound states. Figures 3 and 4 present the LDOS in the middle of the $\mathrm{F}$ region for three domain walls with different widths. In the inset of Fig. 3 we also show the zero-energy LDOS for a domain wall of width $d_{W}=0.2 \xi$ 

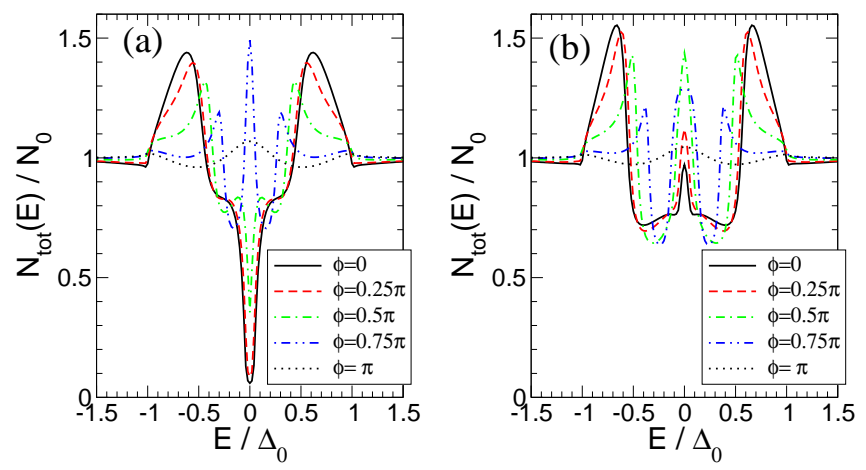

FIG. 4: LDOS as a function of energy for several phase differences $\phi$ between the superconductors, calculated in the middle of $\mathrm{F}$, and for $J=0.5 \Delta_{0}, d_{F}=2.0 \xi$. The width of the domain wall is in (a) $d_{W}=1.0 \xi$, and in (b) $d_{W}=2.0 \xi$.

as a function of the phase difference. As can be seen in

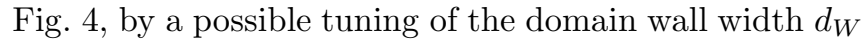
one can always find a region of strongest sensitivity for a given phase difference $\phi$ and vice versa. This increases the possibilities of controlling the zero energy density of states in the domain wall. The rich structure exhibited by these results could easily result in highly nontrivial behavior of the transport current both in equilibrium and nonequilibrium situations.

Conclusions: We have studied numerically the LDOS in a heterostructure consisting of a ferromagnetic alloy sandwiched between two singlet superconductors. We find strong modifications of the LDOS caused by the presence of a domain wall. As only triplet superconducting correlations are sensitive to the direction of the exchange field, the strong variations in the LDOS result from the presence of triplet correlations induced by the spatially varying magnetization. We also find a strong dependence of the density of states in the domain wall on a possible phase difference between the superconducting order parameters, giving an additional tool to control its value. This motivates future studies of the interplay of a supercurrent and the domain wall (Josephson effect). We hope that the variety of features observed in our calculations motivates further experimental research on proximity systems involving weak ferromagnets.

This work was supported by Deutsche Forschungsgemeinschaft within the Center for Functional Nanostructures.
1 A. F. Andreev, Sov. Phys. JETP 19, 1228 (1964).

2 V.G. Kogan, Phys. Rev. B 26, 88 (1982).

3 A. F. Volkov, F. S. Bergeret, and K. B. Efetov, Phys. Rev. Lett. 90, 117006 (2003); F. S. Bergeret, A. F. Volkov, and K. B. Efetov, Phys. Rev. B 69, 174504 (2004); for a recent review see F.S. Bergeret, A.F. Volkov, and K.B. Efetov, cond-mat/0506047 (unpublished).

4 A. Kadigrobov, R.I. Shekhter, and M. Jonson, Europhys. Lett. 54, 394 (2001).

5 Klaus Halterman and Oriol T. Valls, Phys. Rev. B 69, 014517 (2004); Phys. Rev. B 70, 104516 (2004).

6 Z. Radović, N. Lazarides, and N. Flytzanis, Phys. Rev. B 68, 014501 (2003).

7 Ya. V. Fominov, A. A. Golubov, and M. Yu. Kupriyanov, JETP Lett. 77, 510 (2003).

8 Yu. S. Barash, I. V. Bobkova, and T. Kopp, Phys. Rev. B 66, 140503(R) (2002).

9 Daniel Huertas-Hernando, Yu. V. Nazarov, and W. Belzig, Phys. Rev. Lett. 88, 047003 (2002).

10 Jan Petter Morten, Arne Brataas, and Wolfgang Belzig, Phys. Rev. B 72, 014510 (2005).

11 A.I. Buzdin, cond-mat/0505583 (unpublished).

12 T. Kontos et al., Phys. Rev. Lett. 86, 304 (2001).

13 V. V. Ryazanov et al., Phys. Rev. Lett. 86, 2427 (2001); Phys. Rev. B 65, 020501(R) (2001).

14 W. Guichard et al., Phys. Rev. Lett. 90, 167001 (2003).

15 M. Giroud et al., Eur. Phys. J. B 31, 103 (2003).

16 J.Y. Gu et al., Phys. Rev. Lett. 89, 267001 (2002).

17 J. Aumentado and V. Chandrasekhar, Phys. Rev. B 64, 054505 (2001).

18 J.M.E. Geers et al., Phys. Rev. B 64, 094506 (2001).

19 S.M. Frolov et al., Phys. Rev. B 70, 144505 (2004).
20 D. Beckmann, H.B. Weber, and H.v. Löhneysen, Phys. Rev. Lett. 93, 197003 (2004).

21 A. I. Buzdin, L. N. Bulaevskii, and S. V. Panyukov, Pis'ma Zh. Eksp. Teor. Fiz. 35, 147 (1982) [JETP Lett. 35, 178 (1982)].

22 L.N. Bulaevskii et al., Adv. Phys. 34, 175 (1985).

23 F. S. Bergeret, A. F. Volkov, and K. B. Efetov, Phys. Rev. Lett. B 86, 4096 (2001).

24 M. Eschrig et al., Phys. Rev. Lett. 90, 137003 (2003).

25 N. M. Chtchelkatchev and I. S. Burmistrov, Phys. Rev. B 68, 140501(R) (2003).

26 R. Mélin and S. Peysson, Phys. Rev. B 68, 174515 (2003).

27 G. Eilenberger, Z. Phys. 214, 195 (1968).

28 A. I. Larkin and Y. N. Ovchinnikov, Sov. Phys. JETP 28, 1200 (1969).

29 K. D. Usadel, Phys. Rev. Lett. 25, 507 (1977).

30 M. Eschrig, Phys. Rev. B, 61, 9061 (2000).

31 N. Schopohl and K. Maki, Phys. Rev. B 52, 490 (1995); N. Schopohl, cond-mat/9804064 (unpublished).

32 Y. Nagato, K. Nagai and J. Hara, J. Low Temp. Phys. 93, 33 (1993); S. Higashitani and K. Nagai, J. Phys. Soc. Jpn. 64, 549 (1995).

33 M. Eschrig et al., Adv. in Sol. State Phys. 44, pp. 533-546, ed. B. Kramer, Springer Verlag Heidelberg (2004).

34 J.C. Cuevas et al., cond-mat/0507247 (unpublished).

35 T. Löfwander et al., cond-mat/0507398 (unpublished).

36 W. Belzig et al., Superlatt. and Microstruct. 25, 1251 (1999).

37 Yu. V. Nazarov, Superlatt. and Microstruct. 25, 121 (1999) 